\title{
FINITE ELEMENT APPROXIMATION OF SOME DEGENERATE MONOTONE QUASILINEAR ELLIPTIC SYSTEMS*
}

\author{
W. B. LIU ${ }^{\dagger}$ AND JOHN W. BARRETT
}

Abstract. In this paper we examine the continuous piecewise linear finite element approximation of the following system: given $\mathbf{f} \equiv\left(f_{j}\right)$ and $\mathbf{g} \equiv\left(g_{j}\right)$, find $\mathbf{u} \equiv\left(u_{j}\right)(j=1 \rightarrow r$ with $r=1$ or 2$)$ such that

$$
-\nabla \cdot(K(z, \nabla \mathbf{u}(z)) \nabla \mathbf{u}(z))=\mathbf{f}(z), \quad z \in \Omega \subset R^{2},\left.\quad \mathbf{u}\right|_{\partial \Omega}=\left.\mathbf{g}\right|_{\partial \Omega},
$$

where $(\nabla \mathbf{u})_{i j} \equiv \partial u_{j} / \partial z_{i} 1 \leq i \leq 2,1 \leq j \leq r$ and $K$ is a given matrix on $\Omega \times R^{2 \times r}$. We characterize a class of matrices $K$ for which we prove error bounds for this discretization. For sufficiently regular solutions $\mathbf{u}$, achievable at least for some model problems, our bounds improve on existing results in the literature. It is shown that for a notable subclass of $K$, for which only suboptimal error bounds have been previously derived, the piecewise linear finite element approximation of this problem will converge at the optimal rate in an energy-type norm. It is also shown that the techniques used in this paper can be applied to more general problems.

Key words. finite elements, error analysis, degenerate elliptic systems

AMS subject classifications. $65 \mathrm{~N} 30$

1. Introduction. Let $\Omega$ be a bounded open set in $R^{2}$ with a Lipschitz boundary $\partial \Omega$. Let $k(\cdot, \cdot)$ be a positive and continuous function on $\Omega \times(0, \infty)$. Numerous mathematical models from physical processes (see [1], [2], and [6]) have the following form: given $\mathbf{f} \equiv\left(f_{j}\right)$ and $\mathbf{g} \equiv\left(g_{j}\right)$ find $\mathbf{u} \equiv\left(u_{j}\right)(j=1 \rightarrow r$ with $r=1$ or 2$)$ such that

$$
-\nabla \cdot(k(z,|\nabla \mathbf{u}(z)|) \nabla \mathbf{u}(z))=\mathbf{f}(z), \quad z \in \Omega \subset R^{2},\left.\quad \mathbf{u}\right|_{\partial \Omega}=\left.\mathbf{g}\right|_{\partial \Omega},
$$

where $(\nabla \mathbf{u})_{i j} \equiv \partial u_{j} / \partial z_{i} 1 \leq i \leq 2,1 \leq j \leq r$, and $|\cdot|$ denotes the Euclidean norm of a vector or a matrix. The assumptions on the data $\mathbf{f}$ and $\mathbf{g}$ will be specified later. When $k$ is smooth and satisfies the ellipticity and monotonicity conditions given in [11] and [12], there is much work on its well-posedness. Moreover, optimal error bounds for its finite element approximation have been established in various norms. For example in the case $r=1$, if (see [12])

(i) $(z, x) \rightarrow k(z,|x|) x \in C^{2}\left(\bar{\Omega} \times R^{2}\right)^{2}, f \in C^{\alpha}(\bar{\Omega})$ and $g \in C^{2, \alpha}(\bar{\Omega})$ for a $\alpha>0$,

(ii) $\nabla_{x}(k(z,|x|) x)$ is a positive definite matrix for any $z \in \bar{\Omega}$ and $x \in R^{2}$,

(iii) $\int_{\Omega}(k(|\nabla w|) \nabla w-k(|\nabla v|) \nabla v, \nabla(w-v)) d \Omega \geq C_{X} \int_{\Omega}|\nabla(w-v)|^{2} d \Omega$, for any $w, v \in W^{1, \infty}(\Omega)$ such that $(w-v) \in H_{0}^{1}(\Omega)$ and $\|w\|_{W^{1, \infty}(\Omega)}+\|v\|_{W^{1, \infty}(\Omega)} \leq$ $X$, and furthermore if $u \in C^{2, \alpha}(\bar{\Omega})$ and $u^{t}$ (the solution of the deformation equation) is uniformly bounded in $W^{1, \infty}(\Omega)$, then

$$
\left\|u-u^{h}\right\|_{L^{2}(\Omega)} \leq C h^{2} \quad \text { and } \quad\left\|u-u^{h}\right\|_{L^{\infty}(\Omega)} \leq C h^{2}|\ln h|^{3 / 2},
$$

where $u^{h}$ is the piecewise linear finite element approximation of (1.1) based on a quasi-uniform triangulation. In addition, one can deduce by an inverse property that $\left\|u-u^{h}\right\|_{H^{1}(\Omega)} \leq C h$. In [11] the deformation argument was developed further and the same results were obtained for more general systems under weaker assumptions on the data and $u$.

For many physical models $k$ is degenerate; that is, (1.1) is not uniformly elliptic and therefore $k$ does not satisfy these conditions (or the more relaxed conditions in [11]); hence the linearization or deformation procedure used in [11] and [12] cannot be applied at all. A typical example is the $p$-Laplacian, where $k(t) \equiv t^{p-2}$ for a $p \in(1,2) \cup(2, \infty)$. Such models arise in fluid mechanics (see [2] and [7]), nonlinear diffusion (see [18]), and nonlinear

\footnotetext{
*Received by the editors October 28, 1992; accepted for publication (in revised form) April 14, 1994.

†Department of Mathematics, Imperial College, London SW7 2BZ, UK (w.liu@ukc.ac.uk, j.barrett@ic.ac.uk). The research of the first author was supported by SERC grant GR/F81255.
} 
elasticity (see [1] and [19]). For such problems, monotonicity methods play an essential role in establishing well-posedness and the associated error bounds for its finite element approximation (see [8]). The well-posedness of (1.1) has been examined for a class of $k$ in the case $r=1$ by monotonicity methods and in addition error bounds in the energy norm have also been given for the continuous piecewise linear finite element approximation for this class of $k$ (see [4], [6]-[9], [13], [17], and [22]). Due to the limited regularity of solutions to (1.1) in general, there is no advantage in considering higher-order finite element spaces. For many physically interesting problems, most existing bounds are suboptimal, however, (except those in [4]) and may be very poor for some important cases (for example, $k(t) \equiv t^{p-2}$ and $p$ large). On the other hand, numerical computations indicate that the approximation converges at the optimal rate even for such cases at least for sufficiently regular solutions (see [4]). This has been confirmed recently in [4], where optimal error bounds in energy-type norms have been proved for the $p$-Laplacian. It is interesting to find whether this sharper error analysis can be carried out for more general $k$ and how the required regularity on the solution $\mathbf{u}$ varies with the degree of degeneracy in $k$. It is also interesting to examine the finite element approximation of degenerate elliptic systems, not only those of type (1.1), known as Uhlenbeck, where the nonlinearity depends on $|\nabla u|$, but also alternative systems, e.g., with $r=1$. Given $f$ and $g$, find $u$ such that

$$
-\sum_{i=1}^{2} \partial\left(k\left(\left|\partial u / \partial z_{i}\right|\right) \partial u / \partial z_{i}\right) / \partial z_{i}=f \quad \text { in } \Omega,\left.u\right|_{\partial \Omega}=\left.g\right|_{\partial \Omega} .
$$

The above has been extensively studied in the case $k(t) \equiv t^{p-2}$, but there is little work on the finite element error analysis of such problems. We could consider other non-Uhlenbeck type systems (see Remark 4.3). However, for ease of exposition we will consider only problems of type (1.1) and (1.2) in this paper. We will first study them abstractly in a unified framework; later we will see that there are some notable differences between them in obtaining optimal error bounds.

Let $m=1$ or 2 and let $\{I(n)\}(n=1 \rightarrow m)$ be a sequence of positive integers such that $\sum_{n=1}^{m} I(n)=2$. Let $s(0)=0$ and $s(n)=\sum_{i=1}^{n} I(i)$ for $1 \leq n \leq m$. Let $\nabla_{m, n} \mathbf{u}(n=1 \rightarrow m)$ be the $I(n) \times r$ submatrix of $\nabla \mathbf{u}$, defined by the rows from $s(n-1)+1$ to $s(n)$ of $\nabla \mathbf{u}$. We now consider the following system: given $\mathbf{f} \equiv\left(f_{j}\right)$ and $\mathbf{g} \equiv\left(g_{j}\right)$, find $\mathbf{u} \equiv\left(u_{j}\right)(j=1 \rightarrow r$ with $r=1$ or 2 ) such that

$$
-\nabla .(K(z, \nabla \mathbf{u}(z)) \nabla \mathbf{u}(z))=\mathbf{f}(z), \quad z \in \Omega \subset R^{2},\left.\quad \mathbf{u}\right|_{\partial \Omega}=\left.\mathbf{g}\right|_{\partial \Omega},
$$

where $K$ is the block diagonal matrix:

$$
K(z, \nabla \mathbf{u}(z)) \equiv\left(\begin{array}{c}
K_{1}\left(z, \nabla_{m, 1} \mathbf{u}(z)\right) \\
K_{m}\left(z, \nabla_{m, m} \mathbf{u}(z)\right)
\end{array}\right)
$$

and

$$
K_{n}\left(z, \nabla_{m, n} \mathbf{u}(z)\right) \equiv k\left(z,\left|\nabla_{m, n} \mathbf{u}(z)\right|\right) E_{I(n)} \quad \text { for } n=1 \rightarrow m,
$$

where $E_{I(n)}$ is the $I(n) \times I(n)$ unit matrix. If $m=1$, then $I(1)=2$ and $\nabla_{1,1} \mathbf{u} \equiv \nabla \mathbf{u}$ so that we recover the system (1.1). If $m=2$, then $I(1)=I(2)=1, \nabla_{2,1} \mathbf{u} \equiv \partial \mathbf{u} / \partial z_{1}$, and $\nabla_{2,2} \mathbf{u} \equiv \partial \mathbf{u} / \partial z_{2}$ so we have a system of type (1.2). Clearly the problems studied here can be formulated in a simpler way since we have restricted ourselves to the case of two spatial dimensions. The advantages of the above formulation are that it is simple enough to convey the essential difficulties of the problem and our approach and yet it easily generalises 
to accommodate higher-dimensional or more general cases, e.g., if the $k$ appearing in $K_{n}$, $n=1 \rightarrow m$, is not the same. In fact the results in $\S \S 2$ and 3 can easily be generalised to accommodate such cases. Moreover, instead of working with the concrete form (1.3) for $K$, one could state some abstract structural conditions on $K$ in order to prove the error bounds in this paper. Then one only needs to show that (1.1) and (1.2) satisfy these structural conditions. However, in our opinion this approach does not yield any essential benefit unless one can state some good general structural conditions which cover a broad class of degenerate elliptic systems; see Remark 4.3. In addition, we note that it is also important in practice to deal with systems of the form: find $\mathbf{u}$ and $p$ such that

$$
\begin{gathered}
-\nabla .(k(|D(\mathbf{u})|) D(\mathbf{u}))+\nabla p=\mathbf{f} \quad \text { in } \Omega, \\
\nabla . \mathbf{u}=0 \quad \text { in } \Omega,\left.\mathbf{u}\right|_{\partial \Omega}=\left.\mathbf{g}\right|_{\partial \Omega},
\end{gathered}
$$

where $D_{i j}(\mathbf{u}) \equiv(1 / 2)\left(\partial u_{i} / \partial x_{j}+\partial u_{j} / \partial x_{i}\right)$, which arise for example in the mathematical modeling of non-Newtonian flows. The methods presented in this paper serve as a first step in studying such systems (see [3] and [5]).

The purpose of this paper is to extend the error analysis in [4] for the $p$-Laplacian (i.e., $k(t)=t^{p-2}, r=m=1$ ) to the more general class of problems (1.3), and in doing so improve on the best error bounds in the literature [6], where the case $r=m=1$ is studied. It is certainly not a straightforward extension. The first step is to characterise the class of functions $k$ and this cannot be easily deduced from [6]. The conditions on $k$ should be general enough to cover the broadest class of problems, but narrow enough to ensure our sharper error analysis can be applied. In fact, our conditions on $k$ are different from those in [6]. We show how the error bounds and the required regularity on $\mathbf{u}$ for an optimal bound depends on the degree of degeneracy of $k$. The extension to degenerate systems of non-Uhlenbeck type also leads to new difficulties. Furthermore, the analysis in [4] is essentially two-dimensional, whereas the methods used here are readily adaptable to higher-dimensional and more general degenerate systems.

The outline of this paper is as follows: In $\S 2$ we prove some important inequalities and give the system (1.3) a variational formulation. In $\S 3$ abstract error bounds for the continuous piecewise linear finite element approximation are established. Finally, in $\S 4$ explicit error bounds in energy type norms are derived.

Throughout this paper, we adopt the standard notation $W^{v, q}(\Omega)$ for Sobolev spaces on $\Omega$ with norm $\|\cdot\|_{W^{v, q}(\Omega)}$ and semi-norm $|\cdot|_{W^{v, q}(\Omega)}$, and set $H^{\nu}(\Omega) \equiv W^{\nu, 2}(\Omega)$. We note that the semi-norm $|\cdot|_{W^{1, q}(\Omega)}$ and the norm $\|\cdot\|_{W^{1, q}(\Omega)}$ are equivalent on $W_{0}^{1, q}(\Omega)$ and this result is used repeatedly in the paper. In addition, $C$ and $M$ denote two general positive constants independent of $h$ and $z$. For notational convenience we will denote $k(z, t)$ and $K_{n}(z, t)(n=1 \rightarrow m)$ by $k(t)$ and $K_{n}(t)$ for almost every $z \in \Omega$.

2. Variational formulation and some inequalities. In addition to assuming that $k$ is a positive and continuous function on $\Omega \times(0, \infty)$, we also assume that throughout this paper there exists a $p \in(1, \infty)$ such that, for any $t \geq 0$,

$$
k(t) t \leq C\left(1+t^{p-1}\right) .
$$

For ease of exposition, we assume that $\mathbf{g}$ is zero. The results can easily be generalized to the case $\mathbf{g} \not \equiv 0$. Therefore the problem (1.3) has the following weak formulation:

(WP): Find $\mathbf{u} \in \mathbf{V} \equiv\left[W_{0}^{1, p}(\Omega)\right]^{r} \equiv\left\{\mathbf{v} \in\left[W^{1, p}(\Omega)\right]^{r}:\left.\mathbf{v}\right|_{\partial \Omega}=0\right\}$ such that

$$
\int_{\Omega}(K(\nabla \mathbf{u}) \nabla \mathbf{u}, \nabla \mathbf{v}) d \Omega=\int_{\Omega}(\mathbf{f}, \mathbf{v}) d \Omega \quad \forall \mathbf{v} \in \mathbf{V},
$$


where $\mathbf{f} \in \mathbf{V}^{*}$ (the dual space of $\mathbf{V}$ ) and (., .) denotes either the inner product on $R^{2}$ or on real $I \times J$ matrices, where, for $x \equiv\left(x_{i, j}\right)$ and $y \equiv\left(y_{i, j}\right)$, we set $(x, y) \equiv \sum_{j=1}^{J} \sum_{i=1}^{I} x_{i, j} y_{i, j}$ and $|x|^{2} \equiv(x, x)$.

The following minimization problem is associated with (WP):

(MP): Find $\mathbf{u} \in \mathbf{V}$ such that

$$
J_{\Omega}(\mathbf{u})=\operatorname{Min}_{\mathbf{v} \in \mathbf{V}} J_{\Omega}(\mathbf{v})
$$

where

$$
J_{\Omega}(\mathbf{v}) \equiv \sum_{n=1}^{m} \int_{\Omega} \int_{0}^{\left|\nabla_{m, n} \mathbf{v}\right|} k(t) t d t d \Omega-\int_{\Omega}(\mathbf{f}, \mathbf{v}) d \Omega .
$$

It is simple to check that $J_{\Omega}$ is well defined and continuous on $\mathbf{V}$ since

$$
|\nabla \mathbf{v}(z)|^{2} \equiv \sum_{n=1}^{m}\left|\nabla_{m, n} \mathbf{v}(z)\right|^{2} \quad \text { a.e. } z \in \Omega
$$

Furthermore, $J_{\Omega}$ is Gateaux differentiable on $\mathbf{V}$ with

$$
J_{\Omega}^{\prime}(\mathbf{u})(\mathbf{v})=\sum_{n=1}^{m} \int_{\Omega} k\left(\left|\nabla_{m, n} \mathbf{u}\right|\right)\left(\nabla_{m, n} \mathbf{u}, \nabla_{m, n} \mathbf{v}\right) d \Omega-\int_{\Omega}(\mathbf{f}, \mathbf{v}) d \Omega .
$$

In the following, we always assume that $\mathbf{f} \in\left[L^{2}(\Omega)\right]^{r}$.

In the case $r=m=1$ the well-posedness of (WP) and energy-type error bounds for its finite element approximation have been established for a class of functions $k$ which do not generally satisfy the conditions in [11] and [12]. One can refer to, for example, [6]-[9], [13], [17], and [22]. This class has been most completely characterized in [6]. Furthermore, for this class of $k J_{\Omega}$ is strictly convex and coercive on $\mathbf{V}$ so that $J_{\Omega}$ has a unique minimizer on $\mathbf{V}$, which satisfies its Euler equation (WP). In other words the problems (WP) and (MP) are equivalent in this case. In the following we impose an alternative set of assumptions on $k$, which are similar to those in [6], and for this class of functions, we then prove some inequalities which can be viewed as generalizations of Lemmas 1 and 2 in [6]. These play an essential role in the establishment of our improved error bounds over those in [6]. Our conditions are valid in many interesting cases arising in practical problems and appear more convenient to those in [6] for some applications.

In the following all constants are independent of $z \in \Omega$. We now state our assumptions on the function $k$.

Assumption (A): There exist constants $p \in(1,2], \varepsilon, C, M>0$, and $k_{1}, k_{2} \geq 0$ such that

(A1) $k(t) / t^{p-2} \leq C$ for all $t \geq 0$, and $|k(t) t-k(s) s| \leq C|t-s|(s+t)^{p-2}$ for all $t, s>0$ such that $|s / t-1| \leq \varepsilon$.

(A2) $\left(k_{1}+k_{2} t^{2-p}\right) \eta(t) \geq M$, for all $t>0$ where $\eta(t) \in \partial(k(t) t)$ and $\partial(k(t) t)$ is Clarke's generalized gradient of the function $t \rightarrow k(t) t$; see [10].

We note that it follows from (A1) that $t \rightarrow k(t) t$ is locally Lipschitz on $(0, \infty)$ so that the generalized gradient $\partial(k(t) t)$ is well defined for all $t>0$.

Assumption (B): There exist constants $p \in[2, \infty), \varepsilon, C, M>0$, and $k_{1}, k_{2} \geq 0$ such that

(B1) $k(t) /(1+t)^{p-2} \leq C$ for all $t \geq 0$, and $|k(t) t-k(s) s| \leq C|t-s|\left(k_{1}+k_{2}(s+t)^{p-2}\right)$ for all $t, s>0$ such that $|s / t-1| \leq \varepsilon$.

(B2) $t^{2-p} \eta(t) \geq M$ for all $t>0$, where $\eta(t) \in \partial(k(t) t)$.

We now are in the position to state the fundamental inequalities. 
LEMMA 2.1. Let $k$ satisfy (A1) for a $p \in(1,2]$. Then for any $x, y \in R^{I \times J}(I, J \geq 1)$ and $\delta \geq 0$ we have that

$$
|k(|x|) x-k(|y|) y| \leq C|x-y|^{1-\delta}(|x|+|y|)^{p-2+\delta} .
$$

Let $k$ satisfy (A2) for a $p \in(1,2]$. Then for any $x, y \in R^{I \times J}(I, J \geq 1)$ and $\delta \geq 0$ we have that

$$
(k(|x|) x-k(|y|) y, x-y) \geq M|x-y|^{2+\delta}(1+|x|+|y|)^{p-2-\delta} .
$$

Proof. We first prove (2.2a). Some ideas similar to those in [4]-[6], [9], and [13] will be applied. For $x, y \in R^{I \times J}$ let

$$
F(x, y) \equiv|k(|x|) x-k(|y|) y| /\left[|x-y|^{1-\delta}(|x|+|y|)^{p-2+\delta}\right] .
$$

We wish to prove that $F$ is bounded. For $T$ an $I \times I$ orthogonal matrix it follows that for all $(x, y) \in R^{I \times J} \times R^{I \times J}$

$$
F(x, y)=F(y, x), \quad F(T x, T y)=F(x, y),
$$

and

$$
F(0, y) \leq C \quad \text { from (A1). }
$$

Therefore without loss of generality we can suppose that $x, y \neq 0$, in which case

$$
F(x, y) \equiv \frac{\left|\left[k(|x|) x /|x|^{p-1}\right]-\left[k(|y|) y /|y|^{p-1}\right](|y| /|x|)^{p-1}\right|}{|x /| x|-y /| x||^{1-\delta}(1+|y| /|x|)^{p-2+\delta}} .
$$

We can further assume from (2.3a) that $|y| /|x| \leq 1$. To show that $F$ is uniformly bounded it is enough to prove that for any $\eta \geq 0, \varlimsup_{|y /| x|-x /| x|| \rightarrow \eta} F(x, y)<\infty$, where $\eta$ can be infinite. It follows that $F(x, y)$ will be bounded if $|y /| x|-x /| x||$ does not tend to zero as $k(t) / t^{p-2} \leq C$ for $t>0$. Thus it remains to show that $\varlimsup_{y /|x|-e \rightarrow 0} F(x, y)<\infty$ with $e \equiv x /|x|$.

Without loss of generality, assume that there is an $\varepsilon_{0} \in(0, \min (1, \varepsilon))$, where $\varepsilon$ is the constant appearing in (A1), such that ||$y|/| x|-1| \leq \varepsilon_{0}$ and hence $(|x|+|y|)^{2} \leq C|x||y|$ because $|x| /|y|+|y| /|x| \leq 1+\varepsilon_{0}+\left(1-\varepsilon_{0}\right)^{-1}$. Then it follows that, since $|x||y|-(x, y) \leq$ $|x-y|^{2}$,

$$
\begin{aligned}
|k(|x|) x-k(|y|) y|^{2} & =(k(|x|)|x|-k(|y|)|y|)^{2}+2 k(|x|) k(|y|)(|x||y|-(x, y)) \\
& \leq C\left[(|x|-|y|)^{2}(|x|+|y|)^{2(p-2)}+(|x||y|)^{p-2}|x-y|^{2}\right], \\
& \leq C(|x|+|y|)^{2(p-2)}|x-y|^{2} .
\end{aligned}
$$

Consequently, we see that (2.2a) holds with $\delta=0$. In contrast, for any $x, y \in R^{I \times J}$ we have for any $\delta \geq 0$ that $(|x|+|y|)^{p-2}|x-y| \leq(|x|+|y|)^{p-2+\delta}|x-y|^{1-\delta}$; that is, (2.2a) holds for any $\delta \geq 0$.

Similarly, we can prove (2.2b). We first consider the case $\delta=0$. In this case $(2.2 \mathrm{~b})$ follows directly from the proof of Lemma 2 in [6] if one applies the mean value formula for the generalized gradient:

$$
k(t) t-k(s) s=\eta(\lambda t+(1-\lambda) s)(t-s), \quad \text { where } \eta(t) \in \partial(k(t) t) \text { and } \lambda \in(0,1) .
$$

For any $\delta>0$ we have that $|x-y|^{2}(1+|x|+|y|)^{p-2} \geq|x-y|^{2+\delta}(1+|x|+|y|)^{p-2-\delta}$ and so (2.2b) holds for all $\delta \geq 0$. 
LEMMA 2.2. Let $k$ satisfy (B1) for a $p \in[2, \infty)$. Then for any $x, y \in R^{I \times J}(I, J \geq 1)$ and $\delta \geq 0$ we have that

$$
|k(|x|) x-k(|y|) y| \leq C|x-y|^{1-\delta}(1+|x|+|y|)^{p-2+\delta} .
$$

Let $k$ satisfy (B2) for a $p \in[2, \infty)$. Then for any $x, y \in R^{I \times J}(I, J \geq 1)$ and $\delta \geq 0$ we have that

$$
(k(|x|) x-k(|y|) y, x-y) \geq M|x-y|^{2+\delta}(|x|+|y|)^{p-2-\delta} .
$$

Proof. The proof is similar to that of Lemma 2.1 and therefore we present only an outline here. First, we note that we need only to prove (2.4) with $\delta=0$, because the results for $\delta \geq 0$ follow as in the proof of Lemma 2.1. Let

$$
F(x, y) \equiv|k(|x|) x-k(|y|) y| /|x-y|(1+|x|+|y|)^{p-2+\delta}, \quad(x, y) \in R^{I \times J} \times R^{I \times J} .
$$

As before, the key part of the proof is to show that $\overline{\lim }_{y /|x|-e \rightarrow 0} F(x, y)<\infty$, where $e \equiv x /|x|$. We can assume that there is an $\varepsilon_{0} \in(0, \min (1, \varepsilon))$ such that ||$y|/| x|-1| \leq \varepsilon_{0}$. Then from the proof of Lemma 1 in [6] one obtains (2.4a) for $\delta=0$ and hence for all $\delta \geq 0$.

To prove (2.4b) we note that the function $t \rightarrow\left[k(t) t-M t^{p-1} /(p-1)\right]$ is increasing from applying the mean value formula for generalized gradients as used in the proof of Lemma 2.1. Therefore from the proof of Lemma 2 in [6] we have that

$$
(k(|x|) x-k(|y|) y, x-y) \geq M_{1}\left(|x|^{p-2} x-|y|^{p-2} y, x-y\right) .
$$

Without loss of generality we can assume that $|x|>|y|>0$. Let $y=\beta x+\xi$ with $(\xi, x)=0$ so that $|\beta|<1$ as $(y, x)=\beta|x|^{2}$. Let $\mu \equiv\left(|x|^{p-2} x-|y|^{p-2} y, x-y\right)=$ $|x|^{p}+|y|^{p}-\beta\left(|x|^{p-2}+|y|^{p-2}\right)|x|^{2}$. If $4 \beta<1$, then $2 \mu \geq|x|^{p}+|y|^{p} \geq M_{2} \mid x-$ $\left.y\right|^{2}(|x|+|y|)^{p-2}$. On the other hand if $1 \leq 4 \beta<4$, then $\mu=(1-\beta)\left(|x|^{p-2}-\right.$ $\left.|y|^{p-2}\right)|x|^{2}+|x-y|^{2}|y|^{p-2} \geq|x-y|^{2}|y|^{p-2} \geq M_{3}|x-y|^{2}(|x|+|y|)^{p-2}$ as $\beta|x| \leq|y|$. Therefore, in combining the above results we have that (2.4b) holds for $\delta=0$ and hence for all $\delta \geq 0$.

Under similar assumptions, Chow in [6] has proved (2.2a) for $\delta=2-p,(2.2 \mathrm{~b})$ for $\delta=0,(2.4 \mathrm{a})$ for $\delta=0$, and (2.4b) for $\delta=p-2$. With these particular choices of $\delta$ one can establish some error bounds for the finite element approximation of (1.1) (see [6], [9], [13], and [17]). These error bounds, however, are only suboptimal in many important cases. The above generalizations of the inequalities in [6] are crucial in establishing some optimal error bounds in such cases.

The following result is useful in checking (A1) and (B1).

PROPOSITION 2.1. Assume that the function $t \rightarrow k(t) t$ is locally Lipschitz on $(0, \infty)$ and $\eta(t) \in \partial(k(t) t)$. Assumption (A1) for $p \in(1,2]$ and (B1) for $p \in[2, \infty)$ are then equivalent to

(A1) $|\eta(t)| \leq C t^{p-2}$ for all $t>0$.

$(\mathcal{B} 1)|\eta(t)| \leq C\left(k_{1}+k_{2} t^{p-2}\right)$ for all $t>0$.

Proof. As $t \rightarrow k(t) t$ is locally Lipschitz, it follows that it is differentiable at almost every point in $(0, \infty)$. Let $t_{0}$ be a such point. From (A1) it follows that $\left(k\left(t_{0}\right) t_{0}\right)^{\prime} \leq C t_{0}^{p-2}$, and from (B1) that $\left(k\left(t_{0}\right) t_{0}\right)^{\prime} \leq C\left(k_{1}+k_{2} t_{0}^{p-2}\right)$. It then follows from Theorem 2.1 in [10] that (A1) and (B1) imply $(\mathcal{A} 1)$ and $(\mathcal{B} 1)$, respectively. One can also easily infer (A1) and (B1) from $(\mathcal{A} 1)$ and $(\mathcal{B} 1)$, respectively, by applying the mean value formula in [10].

Many functions $k$ met in practical problems (e.g., $k(t) \equiv t^{p-2}, k(t) \equiv\left(1+t^{2}\right)^{(p-2) / 2}$ and $\left.k(t) \equiv[t(1+t)]^{(p-2) / 2}\right)$ indeed satisfy Assumption (A) or (B). Another interesting example from fracture mechanics is the function

$$
k(t) \equiv\left\{\begin{array}{ll}
1 & t \leq 1, \\
t^{p-2} & t \geq 1,
\end{array} \quad \text { with } p \in(1,2] .\right.
$$


(see [19] and [20]). This function is Lipschitz but not differentiable on $(0, \infty)$. It can be shown (see [10]) that

$$
\partial(k(t) t) \equiv \begin{cases}1, & t<1 \\ {[p-1,1],} & t=1 \\ (p-1) t^{p-2}, & t>1\end{cases}
$$

Consequently, from Proposition 2.1 we see that this $k$ satisfies (A). This example shows that the introduction of the generalized gradient in our assumptions is not unnecessary.

Adapting the corresponding proofs in [6] one has the following results.

THEOREM 2.1. Let $k$ satisfy (A) or (B). Given $\mathbf{f} \equiv \mathbf{f}_{i} \in V^{*}$ then (MP) $\equiv(\mathrm{WP})$ has a unique solution $\mathbf{u} \equiv \mathbf{u}_{i} \in \mathbf{V}$. Furthermore, if $p \in(1,2]$

$$
\left\|\mathbf{u}_{1}-\mathbf{u}_{2}\right\|_{\mathbf{v}} \leq C\left[1+\left\|\mathbf{u}_{1}\right\|_{\mathbf{v}}+\left\|\mathbf{u}_{2}\right\|_{\mathbf{v}}\right]^{2-p}\left\|\mathbf{f}_{1}-\mathbf{f}_{2}\right\|_{\mathbf{V}^{*}}
$$

and if $p \in[2, \infty)$

$$
\left\|\mathbf{u}_{1}-\mathbf{u}_{2}\right\|_{\mathbf{V}}^{p-1} \leq C\left\|\mathbf{f}_{1}-\mathbf{f}_{2}\right\|_{\mathbf{V}^{*}} .
$$

Proof. We first note that for any fixed $q>0$ there exist constants $C_{i}(q)$ such that

$$
C_{1} \sum_{n=1}^{m}\left|\nabla_{m, n} \mathbf{v}(z)\right|^{q} \leq|\nabla \mathbf{v}(z)|^{q} \leq C_{2} \sum_{n=1}^{m}\left|\nabla_{m, n} \mathbf{v}(z)\right|^{q} \quad \text { a.e. } z \in \Omega .
$$

It follows from Lemmas 2.1 and 2.2 that $J_{\Omega}$ is a strictly convex and coercive on $\mathbf{V}$ and so (MP) has a unique solution. As (WP) is the Euler equation for (MP), it follows that (MP) $\equiv($ WP). The result (2.6a) follows from Lemma 2.1 with $\delta=0$ and noting from a Holder inequality that

$$
\begin{aligned}
{[1+} & \left.\left\|\nabla \mathbf{u}_{1}\right\|_{\left[L^{p}(\Omega)\right]^{2 \times r}}+\left\|\nabla \mathbf{u}_{2}\right\|_{\left[L^{p}(\Omega)\right]^{2 \times r}}\right]^{p-2}\left\|\nabla\left(\mathbf{u}_{1}-\mathbf{u}_{2}\right)\right\|_{\left[L^{p}(\Omega)\right]^{2 \times r}}^{2} \\
\leq & C \sum_{n=1}^{m}\left\{\left[1+\left\|\nabla_{m, n} \mathbf{u}_{1}\right\|_{\left[L^{p}(\Omega)\right]^{l(n) \times r}}+\left\|\nabla_{m, n} \mathbf{u}_{2}\right\|_{\left[L^{p}(\Omega)\right]^{I(n) \times r}}\right]^{p-2}\right. \\
& \left.\times\left\|\nabla_{m, n}\left(\mathbf{u}_{1}-\mathbf{u}_{2}\right)\right\|_{\left[L^{p}(\Omega)\right]^{I(n) \times r}}^{2}\right\} \\
\leq & C \sum_{n=1}^{m} \int_{\Omega}\left[1+\left|\nabla_{m, n} \mathbf{u}_{1}\right|+\left|\nabla_{m, n} \mathbf{u}_{2}\right|\right]^{p-2}\left|\nabla_{m, n}\left(\mathbf{u}_{1}-\mathbf{u}_{2}\right)\right|^{2} d \Omega .
\end{aligned}
$$

The result (2.6b) follows from Lemma 2.2 with $\delta=p-2$ and noting that

$$
\left\|\nabla\left(\mathbf{u}_{1}-\mathbf{u}_{2}\right)\right\|_{\left[L^{p}(\Omega)\right]^{2 \times r}}^{p} \leq C \sum_{n=1}^{m} \int_{\Omega}\left|\nabla_{m, n}\left(\mathbf{u}_{1}-\mathbf{u}_{2}\right)\right|^{p} d \Omega .
$$

3. Finite element approximation and abstract error bounds. In this section we study the piecewise linear element approximation of (WP). Let $\Omega^{h}$ be a polygonal approximation to $\Omega$ with boundary $\partial \Omega^{h}$. Let $T^{h}$ be a partitioning of $\Omega^{h}$ into disjoint open regular triangles $\tau$, each of maximum diameter bounded above $h$, so that $\bar{\Omega}^{h} \equiv \cup_{\tau \in T^{h}} \bar{\tau}$. We assume that $P_{i} \in \partial \Omega^{h} \Rightarrow P_{i} \in \partial \Omega$ and $\operatorname{dist}\left(\partial \Omega^{h}, \partial \Omega\right) \leq C h^{2}$, where $\left\{P_{i}\right\}$ is the vertex set associated with the triangulation $T^{h}$.

Associated with $T^{h}$ is a finite-dimensional subspace $S^{h}$ of $C\left(\bar{\Omega}^{h}\right)$ such that $\chi_{\mid \tau}$ is linear for all $\chi \in S^{h}$ and $\tau \in T^{h}$. For ease of exposition we will assume that $\Omega^{h} \subseteq \Omega$. Let $V^{h} \equiv\left\{\chi \in S^{h}\right.$ $: \chi\left(P_{i}\right)=0$ for any $\left.P_{i} \in \partial \Omega\right\}$. It follows that $V^{h} \subset W_{0}^{1, p}\left(\Omega^{h}\right)$. Let $\pi_{h}: C\left(\bar{\Omega}^{h}\right) \rightarrow S^{h}$ denote 
the interpolation operator such that for any $v \in C\left(\bar{\Omega}^{h}\right), \pi_{h} v\left(P_{i}\right)=v\left(P_{i}\right) \forall i$. In addition $\pi_{h}:\left[C\left(\bar{\Omega}^{h}\right)\right]^{r} \rightarrow\left[S^{h}\right]^{r}$ is such that for any $\mathbf{v} \in\left[C\left(\bar{\Omega}^{h}\right)\right]^{r}, \pi_{h} \mathbf{v} \equiv\left(\pi_{h} v_{1}, \ldots, \pi_{h} v_{r}\right)$. We recall the following standard approximation results: for $v=0$ or $1, q, s \in[1, \infty]$, and $v \in W^{2, s}(\tau)$ (so that $v \in C(\bar{\tau})$ ), we have that

$$
\left|v-\pi_{h} v\right|_{W^{v, q}(\tau)} \leq C h^{2(1 / q-1 / s)} h^{2-v}|v|_{W^{2, s}(\tau)} \quad \forall \tau \in T^{h}
$$

provided $W^{2, s}(\tau) \subset W^{v, q}(\tau)$. Furthermore if $q>2$, then

$$
\left|v-\pi_{h} v\right|_{W^{v, q}(\tau)} \leq C h^{1-v}|v|_{W^{1, q}(\tau)} \quad \forall \tau \in T^{h} .
$$

We now consider the finite element approximation of (WP) $\equiv(\mathrm{MP})$. A possible finite element approximation of (MP) is the following.

$(\mathrm{MP})^{h}:$ Find $\mathbf{u}^{h} \in \mathbf{V}^{h} \equiv\left[V^{h}\right]^{r}$ such that

$$
J_{\Omega^{h}}\left(\mathbf{u}^{h}\right)=\operatorname{Min}_{\mathbf{v}^{h} \in \mathbf{V}^{h}} J_{\Omega^{h}}\left(\mathbf{v}^{h}\right) .
$$

Equivalently, one can define $\mathbf{u}^{h}$ as the unique solution of the following.

$(\mathrm{WP})^{h}$ : Find $\mathbf{u}^{h} \in \mathbf{V}^{h}$ such that

$$
\sum_{n=1}^{m} \int_{\Omega^{h}} k\left(\left|\nabla_{m, n} \mathbf{u}^{h}\right|\right)\left(\nabla_{m, n} \mathbf{u}^{h}, \nabla_{m, n} \mathbf{v}^{h}\right) d \Omega^{h}=\int_{\Omega^{h}}\left(\mathbf{f}, \mathbf{v}^{h}\right) d \Omega^{h} \quad \forall \mathbf{v}^{h} \in \mathbf{V}^{h} .
$$

The well-posedness of (WP) ${ }^{h}$ and (MP) ${ }^{h}$ follows in an analogous way to that of (WP) and (MP). Furthermore, one can easily establish a discrete analogue of Theorem 2.1. This especially means that for all $h$

$$
\left\|\mathbf{u}^{h}\right\|_{\left[W^{1, p}\left(\Omega^{h}\right)\right]^{r}} \leq C .
$$

Below we establish some abstract error bounds for this approximation. One of the key ideas in our approach is that by using the Lemmas 2.1 and 2.2 we first prove abstract error bounds in a "weighted Sobolev norm," which naturally arises from these degenerate systems.

Let $\mathbf{u}$ be the solution of (WP); then we define for any $\mathbf{v} \in\left[W^{1, p}(\Omega)\right]^{r}$ and for any $\delta \geq-2$

$$
\|\mathbf{v}\|_{(p, \delta)}^{2} \equiv \sum_{n=1}^{m} \int_{\Omega^{h}}\left[\left|\nabla_{m, n} \mathbf{u}\right|+\left|\nabla_{m, n} \mathbf{v}\right|\right]^{p-2-\delta}\left|\nabla_{m, n} \mathbf{v}\right|^{2+\delta} d \Omega^{h}
$$

The above is well defined for all $\mathbf{v} \in\left[W^{1, p}(\Omega)\right]^{r}$ since $\mathbf{u} \in\left[W^{1, p}(\Omega)\right]^{r}$ and

$$
\begin{aligned}
\|\mathbf{v}\|_{(p, \delta)}^{2} & \leq \sum_{n=1}^{m} \int_{\Omega^{h}}\left[\left|\nabla_{m, n} \mathbf{u}\right|+\left|\nabla_{m, n} \mathbf{v}\right|\right]^{p-2-\delta}\left[\left|\nabla_{m, n} \mathbf{u}\right|+\left|\nabla_{m, n} \mathbf{v}\right|\right]^{2+\delta} d \Omega^{h} \\
& \leq C \int_{\Omega^{h}}[|\nabla \mathbf{u}|+|\nabla \mathbf{v}|]^{p} d \Omega^{h}
\end{aligned}
$$

It can be shown that $\|\mathbf{v}\|_{(p, \delta)}^{2}$ is related to a class of quasi-norms. This fact can be very useful, although it is not exploited in this paper.

In establishing these abstract error bounds, it is useful to exploit the fact that $k$ may satisfy (A) not only for a particular $p \in(1,2]$, but also (A1) with $p=p^{+}$for a $p^{+} \in[p, 2]$. Similarly, $k$ may satisfy (B) not only for a particular $p \in[2, \infty)$, but also (B2) with $p=p^{-}$ for a $p^{-} \in[2, p]$. We note that a similar idea has been independently applied in [7] on a particular nondegenerate problem with $p \in(1,2]$ and $p^{+}=2$. 
Example 3.1. Consider for example $k(t)=\left[t^{\mu}(1+t)^{(1-\mu)}\right]^{p-2}$ with $p \in(1, \infty)$ and $\mu \in[0,1]$. If $p \in(1,2]$ then $k$ not only satisfies (A), but also (A1) for any $p^{+} \in[p,(1-$ $\mu) 2+\mu p]$. Similarly, if $p \in[2, \infty)$ then $k$ not only satisfies (B), but also (B2) for any $p^{-} \in[(1-\mu) 2+\mu p, p]$. Clearly for the $p$-Laplacian, $\mu=1$, there is no flexibility in the choice of $p^{+}$and $p^{-}$. However, for $\mu \in[0,1)$ there is flexibility and this will play a crucial role in proving optimal error bounds under minimum regularity requirements.

We can state our abstract error bounds in the following way.

THEOREM 3.1. Let $\mathbf{u}$ and $\mathbf{u}^{h}$ be the unique solutions of (WP) and (WP) $\mathbf{v}^{h} \in \mathbf{V}^{h}$ we have the following.

If $k$ satisfies (A) for a $p \in(1,2]$ and (A1) for a $p^{+} \in[p, 2]$ and if $\mathbf{u} \in\left[W^{1, p^{+}}(\Omega)\right]^{r}$ then for any $\delta_{1} \in(-2,0]$

$$
\left|\mathbf{u}-\mathbf{u}^{h}\right|_{\left[W^{1, p}\left(\Omega^{h}\right)\right]^{r}}^{2} \leq C\left\|\mathbf{u}-\mathbf{v}^{h}\right\|_{\left(p^{+}, \delta_{1}\right)}^{2} .
$$

If $k$ satisfies (B) for a $p \in[2, \infty)$ and (B2) for a $p^{-} \in[2, p]$ then for any $\delta_{2} \geq 0$

$$
\left\|\mathbf{u}-\mathbf{u}^{h}\right\|_{\left(p^{-}, \delta_{2}\right)}^{2} \leq C\left(\left\|\mathbf{v}^{h}\right\|_{\left[W^{1, p}\left(\Omega^{h}\right)\right]^{r}}\right)\left\|\mathbf{u}-\mathbf{v}^{h}\right\|_{\left[W^{1, p}\left(\Omega^{h}\right)\right]^{r}}^{2} ;
$$

and if in addition $\mathbf{u} \in\left[W^{1, \infty}(\Omega)\right]^{r}$ and $\left\|\mathbf{v}^{h}\right\|_{\left[W^{1, \infty}\left(\Omega^{h}\right)\right]^{r}} \leq C$, then for any $q \in[1,2]$

$$
\left\|\mathbf{u}-\mathbf{u}^{h}\right\|_{\left(p^{-}, \delta_{2}\right)}^{2} \leq C\left\|\mathbf{u}-\mathbf{v}^{h}\right\|_{\left[W^{1, q}\left(\Omega^{h}\right)\right\}^{r}}^{q} .
$$

Proof. This proof is similar to that of Theorem 2.1 in [4]. For any $\mathbf{v}^{h} \in \mathbf{V}^{h}$

$$
\begin{aligned}
J_{\Omega^{h}}\left(\mathbf{v}^{h}\right)-J_{\Omega^{h}}(\mathbf{u})= & \int_{0}^{1} J_{\Omega^{h}}^{\prime}\left(\mathbf{u}+s\left(\mathbf{v}^{h}-\mathbf{u}\right)\right)\left(\mathbf{v}^{h}-\mathbf{u}\right) d s \\
= & \int_{0}^{1}\left[J_{\Omega^{h}}^{\prime}\left(\mathbf{u}+s\left(\mathbf{v}^{h}-\mathbf{u}\right)\right)\left(\mathbf{u}+s\left(\mathbf{v}^{h}-\mathbf{u}\right)-\mathbf{u}\right)\right. \\
& \left.\quad-J_{\Omega^{h}}^{\prime}(\mathbf{u})\left(\mathbf{u}+s\left(\mathbf{v}^{h}-\mathbf{u}\right)-\mathbf{u}\right)\right] s^{-1} d s+J_{\Omega^{h}}^{\prime}(\mathbf{u})\left(\mathbf{v}^{h}-\mathbf{u}\right) \\
= & A\left(\mathbf{v}^{h}\right)+J_{\Omega^{h}}^{\prime}(\mathbf{u})\left(\mathbf{v}^{h}-\mathbf{u}\right),
\end{aligned}
$$

where

$$
A\left(\mathbf{v}^{h}\right) \equiv \sum_{n=1}^{m} \int_{0}^{1} \int_{\Omega^{h}} A_{n}\left(\mathbf{v}^{h}\right) d \Omega^{h} d s
$$

and

$$
\begin{aligned}
A_{n}\left(\mathbf{v}^{h}\right) \equiv & \left(k\left(\left|\nabla_{m, n}\left(\mathbf{u}+s\left(\mathbf{v}^{h}-\mathbf{u}\right)\right)\right|\right) \nabla_{m, n}\left(\mathbf{u}+s\left(\mathbf{v}^{h}-\mathbf{u}\right)\right)\right. \\
& \left.-k\left(\left|\nabla_{m, n} \mathbf{u}\right|\right) \nabla_{m, n} \mathbf{u}, \nabla_{m, n}\left(\mathbf{v}^{h}-\mathbf{u}\right)\right) .
\end{aligned}
$$

From $(\mathrm{MP})^{h}$ we have that

$$
\begin{aligned}
& A\left(\mathbf{u}^{h}\right)+J_{\Omega^{h}}^{\prime}(\mathbf{u})\left(\mathbf{u}^{h}-\mathbf{u}\right) \leq J_{\Omega^{h}}\left(\mathbf{u}^{h}\right)-J_{\Omega^{h}}(\mathbf{u}) \leq J_{\Omega^{h}}\left(\mathbf{v}^{h}\right)-J_{\Omega^{h}}(\mathbf{u}) \\
& \quad=A\left(\mathbf{v}^{h}\right)+J_{\Omega^{h}}^{\prime}(\mathbf{u})\left(\mathbf{v}^{h}-\mathbf{u}\right) .
\end{aligned}
$$

Therefore we have that

$$
A\left(\mathbf{u}^{h}\right) \leq A\left(\mathbf{v}^{h}\right)+J_{\Omega^{h}}^{\prime}(\mathbf{u})\left(\mathbf{v}^{h}-\mathbf{u}^{h}\right) .
$$

On the other hand, $-\nabla \cdot(K(\nabla \mathbf{u}) \nabla \mathbf{u})=\mathbf{f} \in\left[L^{2}(\Omega)\right]^{r}$ on $\Omega$ and $\Omega^{h} \subseteq \Omega$, yielding that

$$
J_{\Omega^{h}}^{\prime}(\mathbf{u})\left(\mathbf{v}^{h}-\mathbf{u}^{h}\right)=0 \quad \text { and hence } \quad A\left(\mathbf{u}^{h}\right) \leq A\left(\mathbf{v}^{h}\right) \forall \mathbf{v}^{h} \in \mathbf{V}^{h} .
$$


If $p \in(1,2]$, it follows from $(2.2 \mathrm{a})$ that for all $\delta \in[0,2)$

$$
\begin{aligned}
A\left(\mathbf{v}^{h}\right) \leq & C \sum_{n=1}^{m} \int_{0}^{1} s^{1-\delta} \int_{\Omega^{h}}\left[\left|\nabla_{m, n} \mathbf{u}\right|+\left|\nabla_{m, n}\left(\mathbf{u}+s\left(\mathbf{v}^{h}-\mathbf{u}\right)\right)\right|\right]^{p^{+}-2+\delta} \\
& \times\left|\nabla_{m, n}\left(\mathbf{v}^{h}-\mathbf{u}\right)\right|^{2-\delta} d \Omega^{h} d s \\
\leq & C \sum_{n=1}^{m} \int_{\Omega^{h}}\left[\left|\nabla_{m, n} \mathbf{u}\right|+\left|\nabla_{m, n}\left(\mathbf{v}^{h}-\mathbf{u}\right)\right|\right]^{p^{+}-2+\delta}\left|\nabla_{m, n}\left(\mathbf{v}^{h}-\mathbf{u}\right)\right|^{2-\delta} d \Omega^{h} \\
\equiv & C\left\|\mathbf{u}-\mathbf{v}^{h}\right\|_{\left(p^{+},-\delta\right)}^{2},
\end{aligned}
$$

where we have noted that, for all $\mathbf{v}_{1}, \mathbf{v}_{2}, n=1 \rightarrow m$, and $s \in[0,1]$,

$$
s\left(\left|\nabla_{m, n} \mathbf{v}_{1}\right|+\left|\nabla_{m, n} \mathbf{v}_{2}\right|\right) / 2 \leq\left|\nabla_{m, n}\left(\mathbf{v}_{1}+s \mathbf{v}_{2}\right)\right|+\left|\nabla_{m, n} \mathbf{v}_{1}\right| \leq 2\left(\left|\nabla_{m, n} \mathbf{v}_{1}\right|+\left|\nabla_{m, n} \mathbf{v}_{2}\right|\right) .
$$

Similarly, if $p \in(1,2]$ it follows from $(2.2 \mathrm{~b})$ with $\delta=0$ and (2.8) that

$$
\begin{aligned}
A\left(\mathbf{u}^{h}\right) \geq & M \sum_{n=1}^{M} \int_{0}^{1} s \int_{\Omega^{h}}\left[1+\left|\nabla_{m, n} \mathbf{u}\right|+\left|\nabla_{m, n}\left(\mathbf{u}+s\left(\mathbf{u}^{h}-\mathbf{u}\right)\right)\right|\right]^{p-2} \\
& \times\left|\nabla_{m, n}\left(\mathbf{u}^{h}-\mathbf{u}\right)\right|^{2} d \Omega^{h} d s . \\
\geq & M\left[1+\|\mathbf{u}\|_{\left[W^{1, p}\left(\Omega^{h}\right)\right]^{r}}+\left\|\mathbf{u}^{h}\right\|_{\left[W^{1, p}\left(\Omega^{h}\right)\right]^{r}}\right]^{p-2}\left|\mathbf{u}-\mathbf{u}^{h}\right|_{\left[W^{1, p}\left(\Omega^{h}\right)\right]^{r}}^{2}
\end{aligned}
$$

Combining (3.8) and (3.9) and noting (2.6a) and (3.2) yields the desired result (3.3).

Similarly, if $p \in[2, \infty)$ from (2.4a) with $\delta=0,(2.7)$, and a Holder inequality, we obtain

$$
\begin{aligned}
A\left(\mathbf{v}^{h}\right) \leq & C \sum_{n=1}^{m} \int_{0}^{1} s \int_{\Omega^{h}}\left[1+\left|\nabla_{m, n} \mathbf{u}\right|+\left|\nabla_{m, n}\left(\mathbf{u}+s\left(\mathbf{v}^{h}-\mathbf{u}\right)\right)\right|\right]^{p-2} \\
& \times\left|\nabla_{m, n}\left(\mathbf{v}^{h}-\mathbf{u}\right)\right|^{2} d \Omega^{h} d s \\
\leq & C\left[1+\|\mathbf{u}\|_{\left[W^{1, p}\left(\Omega^{h}\right)\right]^{r}}+\left\|\mathbf{v}^{h}\right\|_{\left[W^{1, p}\left(\Omega^{h}\right)\right]^{r}}\right]^{p-2}\left\|\mathbf{u}-\mathbf{v}^{h}\right\|_{\left[W^{1, p}\left(\Omega^{h}\right)\right]^{r}}^{2} ;
\end{aligned}
$$

from (2.4b) for any $\delta \geq 0$

$$
\begin{aligned}
A\left(\mathbf{u}^{h}\right) \geq & M \sum_{n=1}^{m} \int_{0}^{1} s^{1+\delta} \int_{\Omega^{h}}\left[\left|\nabla_{m, n} \mathbf{u}\right|+\left|\nabla_{m, n}\left(\mathbf{u}+s\left(\mathbf{u}^{h}-\mathbf{u}\right)\right)\right|\right]^{p^{-}-2-\delta} \\
& \times\left|\nabla_{m, n}\left(\mathbf{u}^{h}-\mathbf{u}\right)\right|^{2+\delta} d \Omega^{h} d s \\
\geq & M \sum_{n=1}^{m} \int_{\Omega^{h}}\left[\left|\nabla_{m, n} \mathbf{u}\right|+\left|\nabla_{m, n}\left(\mathbf{u}^{h}-\mathbf{u}\right)\right|\right]^{p^{-}-2-\delta}\left|\nabla_{m, n}\left(\mathbf{u}^{h}-\mathbf{u}\right)\right|^{2+\delta} d \Omega^{h} \\
\equiv & M\left\|\mathbf{u}-\mathbf{u}^{h}\right\|_{\left(p^{-}, \delta\right)}^{2} .
\end{aligned}
$$

Hence the desired result (3.4a) follows, after noting Theorem 2.1 .

If $\mathbf{u} \in\left[W^{1, \infty}(\Omega)\right]^{r}$ and $\left\|\mathbf{v}^{h}\right\|_{\left[W^{1, \delta}\left(\Omega^{h}\right)\right]^{r}}$ is uniformly bounded, from (2.4b) with $\delta=$ $2-q, q \in[1,2]$, and adapting the argument in (3.10a) we obtain that

$$
\begin{aligned}
A\left(\mathbf{v}^{h}\right) \leq & C \sum_{n=1}^{m} \int_{0}^{1} s^{q-1} \int_{\Omega^{h}}\left[1+\left|\nabla_{m, n} \mathbf{u}\right|+\left|\nabla_{m, n}\left(\mathbf{u}+s\left(\mathbf{v}^{h}-\mathbf{u}\right)\right)\right|\right]^{p-q} \\
& \times\left|\nabla_{m, n}\left(\mathbf{v}^{h}-\mathbf{u}\right)\right|^{q} d \Omega^{h} d s \\
\leq & C\left\|\mathbf{u}-\mathbf{v}^{h}\right\|_{\left[W^{1, q}\left(\Omega^{h}\right)\right]^{r}}^{q}
\end{aligned}
$$

Hence we have the desired result (3.4b). 
COROLLARY 3.1. If $k$ satisfies (A) for a $p \in(1,2]$ and (A1) for a $p^{+} \in[p, 2]$ then

$$
\begin{aligned}
\left|\mathbf{u}-\mathbf{u}^{h}\right|_{\left[W^{1, p}\left(\Omega^{h}\right)\right]^{r}} & \leq C\left[\left\|\mathbf{u}-\pi_{h} \mathbf{u}\right\|_{\left[W^{1, p^{+}}\left(\Omega^{h}\right)\right]^{r}}\right]^{p^{+} / 2} \\
& \leq C h^{p^{+} / 2} \quad \text { if } \mathbf{u} \in\left[W^{2, p^{+}}(\Omega)\right]^{r} .
\end{aligned}
$$

If $k$ satisfies (B) for a $p \in[2, \infty)$ and (B2) for a $p^{-} \in[2, p]$ then

$$
\begin{aligned}
\left|\mathbf{u}-\mathbf{u}^{h}\right|_{\left[W^{1, p^{-}}\left(\Omega^{h}\right)\right]^{r}} & \leq C\left(\left\|\pi_{h} \mathbf{u}\right\|_{\left[W^{1, p}\left(\Omega^{h}\right)\right]^{r}}\right)\left[\left\|\mathbf{u}-\pi_{h} \mathbf{u}\right\|_{\left[W^{1, p}\left(\Omega^{h}\right) r^{r}\right.}\right]^{2 / p^{-}} \\
& \leq C h^{2 / p^{-}} \quad \text { if } \mathbf{u} \in\left[W^{2, p}(\Omega)\right]^{r},
\end{aligned}
$$

and

$$
\begin{aligned}
\left|\mathbf{u}-\mathbf{u}^{h}\right|_{\left[W^{1, p^{-}}\left(\Omega^{h}\right)\right]^{r}} & \leq C\left[\left\|\mathbf{u}-\pi_{h} \mathbf{u}\right\|_{\left[W^{1, q}\left(\Omega^{h}\right)\right]^{r}}\right]^{q / p^{-}} \\
& \leq C h^{q / p^{-}} \quad \text { if } \mathbf{u} \in\left[W^{2, q}(\Omega) \cap W^{1, \infty}(\Omega)\right]^{r}, q \in[1,2] .
\end{aligned}
$$

Proof. As $\|\cdot\|_{(q, q-2)}^{2}$ is equivalent to $|\cdot|_{\left[W^{1, q}\left(\Omega^{h}\right) r^{r}\right.}^{q}, q \in(1, \infty)$, choosing $\mathbf{v}^{h} \equiv \pi_{h} \mathbf{u}$, $\delta_{1}=p^{+}-2$ in (3.3), $\delta_{2}=p^{-}-2$ in (3.4), and noting (3.1), one immediately obtains the above error bounds.

Remark 3.1. It follows from the proof of Theorem 3.1 and its corollary that all the above results still hold when the function $k$ in the matrices $K_{n}(n=1 \rightarrow m)$ is not necessarily the same but satisfies assumption (A) or (B) for the same $p$. In addition, one can easily consider more general problems, i.e., systems generated by a linear combination of such $K_{n}$.

Remark 3.2. The best existing results in the literature are those in [6] for $r=m=1$, which correspond to (3.11a) with $p^{+}=p$ and (3.11b) with $p^{-}=p$. Therefore we see that the results (3.11) already improve on these by taking into account the degree of degeneracy in $k$ for a given $p$, i.e., the flexibility in the choice of $p^{+}$and $p^{-}$.

In the next section we explore in more detail the abstract error bounds (3.3) and (3.4), deriving optimal error bounds in many cases.

4. Explicit error bounds. In this section we deduce explicit error bounds from the abstract bounds derived in the previous section. We first consider the simplest case of $k$ satisfying (A) for a $p \in(1,2]$ and (A1) for $p^{+}=2$; that is, $t \rightarrow k(t) t$ is globally Lipschitz on $[0, \infty)$, i.e., $k$ is nondegenerate. A similar result has recently appeared in [7] in the case $r=m=1$.

THEOREM 4.1. Let $k$ satisfy (A) for a $p \in(1,2]$ and (A1) for $p^{+}=2$. Let $\mathbf{u}$ and $\mathbf{u}^{h}$ be the unique solutions of (WP) and (WP $)^{h}$. If $\mathbf{u} \in\left[H^{2}(\Omega)\right]^{r}$, then

$$
\left\|\mathbf{u}-\mathbf{u}^{h}\right\|_{\left[W^{1, p}\left(\Omega^{h}\right)\right]^{r}}^{2} \leq C h^{2} .
$$

Proof. First we note from a Poincare inequality that if $\mathbf{v} \in\left[W^{1, q}\left(\Omega^{h}\right)\right]^{r}, q \in(1, \infty)$, and $\mathbf{v}^{h}, \mathbf{w}^{h} \in \mathbf{V}^{h}$ then

$$
\left\|\mathbf{v}-\mathbf{v}^{h}\right\|_{\left[W^{1, q}\left(\Omega^{h}\right)\right]^{r}} \leq\left\|\mathbf{v}-\mathbf{w}^{h}\right\|_{\left[W^{1, q}\left(\Omega^{h}\right)\right]^{r}}+\left|\mathbf{v}-\mathbf{v}^{h}\right|_{\left[W^{1, q}\left(\Omega^{h}\right)\right]^{r}} .
$$

The desired result then follows directly from (3.11a) and (4.1) with $q=p, \mathbf{v} \equiv \mathbf{u}, \mathbf{v}^{h} \equiv \mathbf{u}^{h}$, and $\mathbf{w}^{h} \equiv \pi_{h} \mathbf{u}$.

Example 4.1. Let $k(t)=\left(1+t^{2}\right)^{(p-2) / 2}$ with $p \in(1,2]$. Then $k$ satisfies the assumptions of Theorem 4.1 and $\mathbf{u}^{h}$ will converge to $\mathbf{u}$ at the optimal rate $h$ in $\left[W^{1, p}\left(\Omega^{h}\right)\right]^{r}$, provided $\mathbf{u} \in\left[H^{2}(\Omega)\right]^{r}$. This regularity requirement has recently been proven achievable for a class of functions $k$, including the above choice, in the case $r=m=1$ if $\Omega$ is convex or $C^{2}$ and $f \in L^{q}(\Omega), q>2$ (see [14]). This error bound is, of course, an improvement over the 
suboptimal error bound, $C h^{p / 2}$, given in [6] for the case $r=m=1$. We note that one can apply the theory in [12] and [11] to this case and obtain the optimal rate given above under a stronger regularity assumption on $\mathbf{u}$ and if the triangulation is quasi-uniform. However, Theorem 4.1 can be applied to more general cases such as $k(t)$ defined by (2.5), where the theory in [12] and [11] does not apply.

Before we consider improved error bounds for degenerate $k$, we first make some remarks about the regularity of solutions in the simplest case $(r=m=1)$.

Remark 4.1. Consider the case $r=m=1$ and $k(t)=t^{p-2}, p \in(1, \infty)$. It has been proven that $u \in C^{1, \beta}(\bar{\Omega})$ for a $\beta>0$ if $f \in L^{\infty}(\Omega)$ and $\partial \Omega \in C^{2}$ (see [21]). However, there do not appear to be any regularity results in the literature which can be used to derive explicit finite element error bounds. All the results are either first-order or local in character except the recent results in [14]-[16]. Below we consider some model problems in order to gain some insight into the regularity of solutions in general.

Example 4.2. Let $k(t)=t^{p-2}, p \in(1, \infty)$, and $r=m=1$. Consider the onedimensional version of (WP) with $\Omega=(-1,1)$ and $u(-1)=u(1)=0$. It is easy to establish the following results (see [4]).

$$
p \in(1,2) \text { then } f \in L^{2}(\Omega) \Rightarrow u \in H^{2}(\Omega) \text { and } f \in W^{1,1}(\Omega) \Rightarrow u \in W^{3,1}(\Omega),
$$

$$
\begin{aligned}
& p \in(1,3 / 2] \text { and } f \in C^{0, \beta}(\bar{\Omega}) \text { for } \beta \in[0,1] \Rightarrow u \in C^{2, \beta}(\bar{\Omega}), \\
& p \in[3 / 2,2) \text { and } f \in C^{0, \beta}(\bar{\Omega}) \text { for } \beta \in[0,(2-p) /(p-1)] \Rightarrow u \in C^{2, \beta}(\bar{\Omega}), \\
& \quad p \in(2, \infty) \text { and } f \in L^{\infty}(\Omega) \Rightarrow u \in C^{1,1 /(p-1)}(\bar{\Omega}), \\
& p \in(2, \infty) \text { and } \\
& f \in C(\bar{\Omega}) \text { changes sign only a finite number of times } \Rightarrow u \in W^{2,1}(\Omega) .
\end{aligned}
$$

By considering the specific case of $f \equiv 1$, yielding

$$
u(z)=(p-1)\left(1-|z|^{p /(p-1)}\right) / p,
$$

we see that the results $(4.2 \mathrm{c}-\mathrm{d})$ are sharp. In addition, we see that

$$
\begin{gathered}
p \in[3 / 2,2) \Rightarrow u \in W^{3, q}(\Omega) \quad \text { if } q<(p-1) /(2 p-3), \\
p>2 \Rightarrow u \in W^{2, q}(\Omega) \quad \text { if } q<(p-1) /(p-2),
\end{gathered}
$$

and therefore the sharpness of the second result given in (4.2a) as $p \rightarrow 2$ and (4.2e) as $p \rightarrow \infty$.

We now consider the radially symmetric case with $\Omega \equiv\{(r, \theta): r<1\}$ and $f(z) \equiv$ $F(r)$. Once again the following results are easily established (see [4]). The results $(4.2 \mathrm{~b}-\mathrm{e})$ still hold and in place of (4.2a) we have that

$$
\begin{aligned}
& p \in(1,2) \text { then } f \in L^{q}(\Omega) \text { for } q>2 \Rightarrow u \in H^{2}(\Omega) \text { and } \\
& f \in W^{1, q}(\Omega) \text { for } q>1 \Rightarrow u \in W^{3,1}(\Omega) .
\end{aligned}
$$

By considering the specific example of $f \equiv 1$ and $g=0$, yielding

$$
u(r)=C\left(1-r^{p /(p-1)}\right),
$$

we see the sharpness of $(4.2 c-d)$ once again. However, we see that $(4.5 a)$ is such that

$$
p \in(1,3 / 2] \Rightarrow C^{3}(\bar{\Omega}) \quad \text { at least, }
$$




$$
\begin{gathered}
p \in(3 / 2,2) \Rightarrow u \in W^{3, s}(\Omega) \quad \text { if } s<2(p-1) /(2 p-3), \\
p>2 \Rightarrow u \in W^{2, s}(\Omega) \quad \text { if } s<2(p-1) /(p-2),
\end{gathered}
$$

and therefore the second result given in (4.4) and (4.2e) may not be sharp. To prove higher-order global regularity of $u$ for a general two-dimensional domain is very difficult. Nevertheless, it has been shown in $[16]$ that $C^{2, \mu}(\bar{\Omega})$ and $W^{3, p}(\Omega)$ regularity, which is required in Theorems $4.2 \mathrm{a}$ and $4.2 \mathrm{~b}$ in the case $p \in(1,2]$, can indeed be achieved for a class of $k$ satisfying assumptions similar to (A) or (B) if $\partial \Omega \in C^{2,1}, f \equiv 0$, and, for a restricted class of Dirichlet data, $g$ (see [16]). We should add that we know of no counterexample to this required regularity for general smooth data $\partial \Omega, f$ and $g$.

We now improve on the error bound (3.11a) when $k$ satisfies (A). As stated earlier, the best existing error bound in the literature for $p \in(1,2]$ and $r=m=1$ is $\left\|u-u^{h}\right\|_{W^{1, p}\left(\Omega^{h}\right)}^{2} \leq C h^{p}$ if $u \in W^{2, p}(\Omega)$ (see [6]). Below we prove an optimal rate of convergence, replacing $h^{p}$ by $h^{2}$ in the above, under a stronger regularity requirement on $u$. We start with a lemma.

LEMMA 4.1. Let $\mathbf{w} \in\left[W^{2,1}(D)\right]^{I \times r}$ with $1 \leq I, r \leq 2$, where $D$ is a bounded open set in $R^{2}$ with a Lipschitz boundary $\partial D$. Then for $\alpha \in(-1,0]$

$$
\int_{D}|\mathbf{w}|^{\alpha}|\nabla \mathbf{w}|^{2} d D<\infty
$$

Proof. Let $\mathbf{w} \equiv\left(w_{i, j}\right)$ and so

$$
|\nabla \mathbf{w}|^{2} \equiv \sum_{i=1}^{I} \sum_{j=1}^{r} \sum_{k=1}^{2}\left(\partial w_{i, j} / \partial z_{k}\right)^{2}
$$

It follows that

$$
\int_{D}|\mathbf{w}|^{\alpha}|\nabla \mathbf{w}|^{2} d D \leq C \sum_{i=1}^{I} \sum_{j=1}^{r} \int_{D}\left|w_{i, j}\right|^{\alpha} \sum_{k=1}^{2}\left(\partial w_{i, j} / \partial z_{k}\right)^{2} d D .
$$

By Green's formula, with $\left(v_{1}, v_{2}\right)^{T}$ the outward unit normal of $\partial D$, we have that, for $1 \leq i \leq I$ and $1 \leq j \leq r$,

$$
\begin{aligned}
(\alpha+1) & \int_{D}\left|w_{i, j}\right|^{\alpha} \sum_{k=1}^{2}\left(\partial w_{i, j} / \partial z_{k}\right)^{2} d D \\
= & \int_{\partial D} \operatorname{sign}\left(w_{i, j}\right)\left[\sum_{k=1}^{2} v_{k}\left(\partial w_{i, j} / \partial z_{k}\right)\right]\left|w_{i, j}\right|^{\alpha+1} d s \\
& -\int_{D} \operatorname{sign}\left(w_{i, j}\right) \Delta w_{i, j}\left|w_{i, j}\right|^{\alpha+1} d D \\
\leq & C\left\|w_{i, j}\right\|_{C(\bar{D})}^{\alpha+1}\left\|W_{i, j}\right\|_{W^{2,1}(D)}<\infty \quad \text { as } w_{i, j} \in W^{2,1}(D),
\end{aligned}
$$

where we have noted the imbedding $W^{2,1}(D) \subset C(\bar{D})$ and a trace inequality. Hence we have the desired result (4.6).

THEOREM 4.2a. Let $k$ satisfy (A) for $a p \in(1,2)$ and (A1) for $a p^{+} \in[p, 2)$. Let $\mathbf{u}$ and $\mathbf{u}^{h}$ be the unique solutions of (WP) and (WP) ${ }^{h}$ with $m=1$. If

$$
\int_{\Omega}|\nabla \mathbf{u}|^{p^{+}-2}|H[\mathbf{u}]|^{2} d \Omega<\infty
$$


where $H[\mathbf{u}] \equiv\left(H\left[u_{1}\right], \ldots, H\left[u_{r}\right]\right)$ and $H\left[u_{j}\right] \equiv \nabla\left(\nabla u_{j}\right)(1 \leq j \leq r)$ is the Hessian matrix of $u_{j}$, and $\mathbf{u} \in\left[C^{2, \beta}(\bar{\Omega})\right]^{r}$ for a $\beta>0$, then

$$
\left\|\mathbf{u}-\mathbf{u}^{h}\right\|_{\left[W^{1, p}\left(\Omega^{h}\right)\right]^{r}}^{2} \leq C\left[h^{2}+h^{p^{+}(1+\beta)}\right] .
$$

Hence if $\mathbf{u} \in\left[C^{2,2 / p^{+}-1}(\bar{\Omega}) \cap W^{3,1}(\Omega)\right]^{r}$, then

$$
\left\|\mathbf{u}-\mathbf{u}^{h}\right\|_{\left[W^{1, p}\left(\Omega^{h}\right)\right]^{r}} \leq C h .
$$

Proof. For $\mathbf{u} \in\left[W^{2, \infty}(\Omega)\right]^{r}$, we have from (3.1) that for all $\tau_{j} \in T^{h}$ there exists a $z^{(j)} \in \bar{\tau}_{j}$ such that for all $z \in \bar{\tau}_{j}$

$$
\left|\nabla\left(\mathbf{u}-\pi_{h} \mathbf{u}\right)(z)\right| \leq C h\left|H[\mathbf{u}]\left(z^{(j)}\right)\right|
$$

If in addition $\mathbf{u} \in\left[C^{2, \beta}(\bar{\Omega})\right]^{r}$, we have that

$$
\left|\nabla\left(\mathbf{u}-\pi_{h} \mathbf{u}\right)(z)\right| \leq C h|H[\mathbf{u}](z)|+C h^{1+\beta} .
$$

Furthermore, it is easy to check that the function $q(t)=(a+t)^{\alpha} t^{2}$ with $a \geq 0$ and $\alpha \in(-1,0]$ is increasing on $R^{+}$and $q\left(\left|t_{1}+t_{2}\right|\right) \leq 2\left[q\left(\left|t_{1}\right|\right)+q\left(\left|t_{2}\right|\right)\right]$ for all $t_{1}, t_{2} \in R$. Therefore from (3.3) with $\delta_{1}=0$ it follows that

$$
\begin{aligned}
\left|\mathbf{u}-\mathbf{u}^{h}\right|_{\left[W^{1, p}\left(\Omega^{h}\right)\right]^{r}}^{2} & \leq C \int_{\Omega^{h}}\left[|\nabla \mathbf{u}|+\left|\nabla\left(\mathbf{u}-\pi_{h} \mathbf{u}\right)\right|\right]^{p^{+}-2}\left|\nabla\left(\mathbf{u}-\pi_{h} \mathbf{u}\right)\right|^{2} d \Omega^{h} \\
& \leq C h^{2} \int_{\Omega^{h}}|\nabla \mathbf{u}|^{p^{+}-2}|H[\mathbf{u}]|^{2} d \Omega^{h}+C h^{p^{+}(1+\beta)}
\end{aligned}
$$

The desired result (4.8a) then follows from (4.7) and (4.1). The result (4.8b) follows immediately by noting Lemma 4.1 with $\alpha=p^{+}-2, \mathbf{w} \equiv \nabla \mathbf{u}$, and hence $\nabla \mathbf{w} \equiv H[\mathbf{u}]$.

It is worth noting that the condition (4.7) actually holds in the case $r=1$ if $f \in L^{\infty}(\Omega)$ and $\partial \Omega \in C^{2}$ (see [15] and [21]). Thus, in this case we only require that $u \in C^{2,2 / p^{+}-1}(\bar{\Omega})$. Because it may be difficult in practice to establish that $\mathbf{u} \in\left[C^{2, \beta}(\bar{\Omega})\right]^{r}$, the following result may be more useful in some cases.

THEOREM 4.2b. Let $k$ satisfy (A) for a $p \in(1,2)$. Let $\mathbf{u}$ and $\mathbf{u}^{h}$ be the unique solutions of (WP) and (WP) $)^{h}$ with $m=1$. If $\mathbf{u} \in\left[W^{3, p}(\Omega)\right]^{r}$, then

$$
\left\|\mathbf{u}-\mathbf{u}^{h}\right\|_{\left[W^{1, p}\left(\Omega^{h}\right)\right]^{r}} \leq C h .
$$

Proof. Given $\mathbf{u} \in\left[W^{3, p}(\Omega)\right]^{r}$, we introduce $\left\{\mathbf{u}^{(i)}\right\}_{i=1}^{\infty}$ with $\mathbf{u}^{(i)} \in\left[C^{3}(\bar{\Omega})\right]^{r}$ for all $i$ and $\mathbf{u}^{(i)} \rightarrow \mathbf{u}$ strongly in $\left[W^{3, p}(\Omega)\right]^{r}$ as $i \rightarrow \infty$. We have from (3.1) that for all $\tau_{j} \in T^{h}$ there exists a $z^{(j)}(i) \in \bar{\tau}_{j}$ such that for all $z \in \bar{\tau}_{j}$

$$
\begin{aligned}
\left|\nabla\left(\mathbf{u}^{(i)}-\pi_{h} \mathbf{u}^{(i)}\right)(z)\right| & \leq C h\left|H\left[\mathbf{u}^{(i)}\right]\left(z^{(j)}\right)\right| \\
& \leq C h\left|H\left[\mathbf{u}^{(i)}\right](z)\right|+C h\left|H\left[\mathbf{u}^{(i)}\right]\left(z^{(j)}\right)-H\left[\mathbf{u}^{(i)}\right](z)\right|,
\end{aligned}
$$


where $H\left[\mathbf{u}^{(i)}\right]$ is as defined in Theorem 4.2a. A similar calculation to that in Theorem 4.2a yields

$$
\begin{aligned}
& \int_{\Omega^{h}}\left[\left|\nabla \mathbf{u}^{(i)}\right|+\left|\nabla\left(\mathbf{u}^{(i)}-\pi_{h} \mathbf{u}^{(i)}\right)\right|\right]^{p-2}\left|\nabla\left(\mathbf{u}^{(i)}-\pi_{h} \mathbf{u}^{(i)}\right)\right|^{2} d \Omega^{h} \\
& \leq C h^{2} \int_{\Omega^{h}}\left|\nabla \mathbf{u}^{(i)}\right|^{p-2}\left|H\left[\mathbf{u}^{(i)}\right]\right|^{2} d \Omega^{h} \\
& \quad+C h^{2+p} \sum_{j} \int_{\tau_{j}}\left|H\left[\mathbf{u}^{(i)}\right]\left(z^{(j)}\right)-H\left[\mathbf{u}^{(i)}\right](z)\right|^{p} d z \\
& \leq C h^{2} \int_{\Omega^{h}}\left|\nabla \mathbf{u}^{(i)}\right|^{p-2}\left|H\left[\mathbf{u}^{(i)}\right]\right|^{2} d \Omega^{h} C h^{2(p+1)} \int_{\Omega^{h}}\left|\nabla H\left[\mathbf{u}^{(i)}\right]\right|^{p} d \Omega^{h} \\
& \leq C\left(\left\|\mathbf{u}^{(i)}\right\|_{\left[W^{3, p}(\Omega)\right]^{r}}\right) h^{2},
\end{aligned}
$$

where we have applied Lemma 4.1 with $\alpha=p-2$ and $\mathbf{w} \equiv \nabla \mathbf{u}^{(i)}$.

Before proceeding further we note the following results. If $q_{1}(t) \equiv(|\nabla \mathbf{u}|+t)^{p-2} t^{2}$ and $q_{2}(t) \equiv\left|\nabla\left(\mathbf{u}^{(i)}-\pi_{h} \mathbf{u}^{(i)}\right)\right|^{2}\left(\left|\nabla\left(\mathbf{u}^{(i)}-\pi_{h} \mathbf{u}^{(i)}\right)\right|+t\right)^{p-2}$, then it follows for $a, b \geq 0$ that

$$
\left|q_{1}(a)-q_{1}(b)\right| \leq 2(|\nabla \mathbf{u}|+a+b)^{p-1}|a-b|
$$

and

$$
\left|q_{2}(a)-q_{2}(b)\right| \leq\left|\nabla\left(\mathbf{u}^{(i)}-\pi_{h} \mathbf{u}^{(i)}\right)\right|^{p-1}|a-b| .
$$

From (3.1b) it follows that $\left|\pi_{h} \mathbf{v}\right|_{\left[W^{1, \infty}\left(\Omega^{h}\right)\right]^{r}} \leq C|\mathbf{v}|_{\left[W^{1, \infty}\left(\Omega^{h}\right)\right]^{r}}$.

From (3.3) with $\delta_{1}=0$ and $p^{+}=p,(4.10)$, and (4.11) it follows that

$$
\begin{aligned}
& \left|\mathbf{u}-\mathbf{u}^{h}\right|_{\left[W^{1, p}\left(\Omega^{h}\right)\right]^{r}}^{2} \leq C\left\|\mathbf{u}-\pi_{h} \mathbf{u}\right\|_{(p, 0)}^{2} \\
& \leq \int_{\Omega^{h}}\left[q_{2}\left(\left|\nabla \mathbf{u}^{(i)}\right|\right)+\left|q_{2}(|\nabla \mathbf{u}|)-q_{2}\left(\left|\nabla \mathbf{u}^{(i)}\right|\right)\right|\right] d \Omega^{h} \\
& \quad+\int_{\Omega^{h}}\left|q_{1}\left(\left|\nabla\left(\mathbf{u}-\pi_{h} \mathbf{u}\right)\right|\right)-q_{1}\left(\left|\nabla\left(\mathbf{u}^{(i)}-\pi_{h} \mathbf{u}^{(i)}\right)\right|\right)\right| d \Omega^{h} \\
& \leq C\left(\left\|\mathbf{u}^{(i)}\right\|_{\left[W^{3, p}(\Omega)\right]^{r}}\right) h^{2} \\
& \quad+C\left[|\mathbf{u}|_{\left[W^{1, \infty}(\Omega)\right]^{r}}+\left|\mathbf{u}^{(i)}\right|_{\left[W^{1, \infty}(\Omega)\right]^{r}}\right]^{p-1}\left|\left(\mathbf{u}-\mathbf{u}^{(i)}\right)\right|_{\left[W^{1,1}(\Omega)\right]^{r}} .
\end{aligned}
$$

Because the right-hand side of the above $\rightarrow C\left(\|\mathbf{u}\|_{\left[W^{3, p}(\Omega)\right]^{r}}\right) h^{2}$ as $i \rightarrow \infty$, the desired result (4.9) follows after noting (4.1).

From Example 4.2 we see that the regularity requirement $\mathbf{u} \in\left[C^{2,2 / p^{+}-1}(\bar{\Omega}) \cap W^{3,1}(\Omega)\right]^{r}$ in Theorem 4.2a to guarantee that $\mathbf{u}^{h}$ converges to $\mathbf{u}$ at the optimal rate $h$ in $\left[W^{1, p}\left(\Omega^{h}\right)\right]^{r}$ as $h \rightarrow 0$ (see (4.8b)) is achieved in both the one-dimensional and radially symmetric model problems for $f$ sufficiently smooth. It is also achieved in some physically relevant cases discussed in [16] for general $k$ and for general two-dimensional domains as mentioned in Example 4.2. Whereas (4.3b) suggests that Theorem $4.2 \mathrm{~b}$ is not generally applicable in one dimension as this regularity requirement, $u \in W^{3, p}(\Omega)$ is not achieved even on this very simple problem. However, from $(4.5 b-c)$ we see that it is applicable to the corresponding radially symmetric case. If, in addition, we assume that $f$ is piecewise Holder continuous, then it can be shown that the regularity requirement can be weakened to $\mathbf{u} \in\left[W^{2 / p^{+}+1, p^{+}}(\Omega)\right]^{r}$ in order to prove an optimal rate of convergence in $\left[W^{1, p}\left(\Omega^{h}\right)\right]^{r}$. We consider this to be the 
best possible result since it collapses to $\mathbf{u} \in\left[H^{2}(\Omega)\right]^{r}$ for $p^{+}=2$. These regularity issues are addressed in [16].

Remark 4.2. The above results also demonstrate how the regularity requirement on $\mathbf{u}$, in order to obtain an optimal error bound, increases as the degeneracy in $k$ increases. For example, let $r=1, \partial \Omega \in C^{2}$, with either (i) $f \in L^{\infty}(\Omega)$ or (ii) $f$ piecewise Holder continuous. Now either (i) $C^{2,2 / p^{+}-1}(\bar{\Omega})$ or (ii) $W^{2 / p^{+}+1, p^{+}}(\Omega)$ regularity is required on $u$ in order to obtain (4.8b). Consider for example $k(t)=\left[t^{\mu}(1+t)^{(1-\mu)}\right]^{p-2}$ with $p \in(1,2]$ and $\mu \in[0,1]$. Then, as previously noted in Example 3.1, we can choose $p^{+}=2+\mu(p-2)$. Therefore, the regularity requirement on $u$ increases as the degree of degeneracy in $k,|\mu(p-2)|$, increases.

We now consider the case $m=2$. In order to prove an optimal error we need to show that $\left\|\mathbf{u}-\pi_{h} \mathbf{u}\right\|_{\left(p^{+}, 0\right)}^{2} \leq C h^{2}$. This is, however, not necessarily true in this case, as can be seen from the following example.

Example 4.3. Consider the function $u\left(z_{1}, z_{2}\right)=z_{2}^{4}$, which is a solution of (1.2) for $k(t) \equiv t^{p-2}, p \in(1,2]$ with $f \in C(\bar{\Omega})$ and $g$ smooth. It is easy to see in this case that

$$
\left\|u-\pi_{h} u\right\|_{\left(p^{+}, 0\right)}^{2} \geq \int_{\Omega^{h}}\left|\partial\left(u-\pi_{h} u\right) / \partial z_{1}\right|^{p^{+}} d \Omega^{h},
$$

as $\partial u / \partial z_{1}=0$ in $\Omega$. Thus in general $\left\|u-\pi_{h} u\right\|_{\left(p^{+}, 0\right)}^{2}$ is only order $h^{p^{+}}$.

However, if the triangulation $T^{h}$ is such that every $\tau \in T^{h}$ has two of its sides parallel to the $z_{1}$ and $z_{2}$ axes, then one can easily establish that $\partial\left(u-\pi_{h} u\right) / \partial z_{1}=0$ and, moreover, show that $\left\|u-\pi_{h} u\right\|_{\left(p^{+}, 0\right)}^{2} \leq C h^{2}$. This restriction is not too severe, since such triangulations are commonly used in practice.

THEOREM 4.3. Let $k$ satisfy (A) for a $p \in(1,2)$ and (A1) for a $p^{+} \in[p, 2)$. Let $\mathbf{u}$ and $\mathbf{u}^{h}$ be the unique solutions of $(\mathrm{WP})$ and $(W P)^{h}$ with $m=2$. Assume that the triangulation $T^{h}$ of $\Omega^{h}$ is such that every $\tau \in T^{h}$ has two sides parallel to the $z_{1}$ and $z_{2}$ axes. If $\mathbf{u} \in\left[C^{2, \beta}(\bar{\Omega})\right]^{r}$ for $a \beta>0$ and

$$
\sum_{n=1}^{2} \int_{\Omega}\left|\nabla_{2, n} \mathbf{u}\right|^{p^{+}-2}\left|H_{2, n}[\mathbf{u}]\right|^{2} d \Omega<\infty,
$$

where $H_{m, n}[\mathbf{u}] \equiv\left(H_{m, n}\left[u_{1}\right], \ldots, H_{m, n}\left[u_{r}\right]\right)$ and $H_{m, n}\left[u_{j}\right] \equiv \nabla\left(\nabla_{m, n} u_{j}\right)(1 \leq j \leq r$ and $1 \leq$ $n \leq m)$ is the submatrix of Hessian matrix of $u_{j}$ (rows $s(n-1)+1$ to $\left.s(n)\right)$, then

$$
\left\|\mathbf{u}-\mathbf{u}^{h}\right\|_{\left[W^{1, p}\left(\Omega^{h}\right)\right]^{r}}^{2} \leq C\left[h^{2}+h^{p^{+}(1+\beta)}\right] .
$$

Hence if $\mathbf{u} \in\left[C^{2,2 / p^{+}-1}(\bar{\Omega}) \cap W^{3,1}(\Omega)\right]^{r}$, then

$$
\left\|\mathbf{u}-\mathbf{u}^{h}\right\|_{\left[W^{1, p}\left(\Omega^{h}\right)\right]^{r}} \leq C h .
$$

Proof. For $\mathbf{u} \in\left[W^{2, \infty}(\Omega)\right]^{r}$, we have from (3.1) that for all $\tau_{j} \in T^{h}$ there exists a $z^{(j)}(n) \in \bar{\tau}_{j}$ such that for all $z \in \bar{\tau}$

$$
\left|\nabla_{2, n}\left(\mathbf{u}-\pi_{h} \mathbf{u}\right)(z)\right| \leq C h\left|H_{2, n}[\mathbf{u}]\left(z^{(j)}(n)\right)\right|, \quad n=1 \rightarrow 2 .
$$

To see this let us note that for $i=1 \rightarrow r, \nabla_{2, n}\left(u_{i}-\pi_{h} u_{i}\right) \equiv \partial\left(u_{i}-\pi_{h} u_{i}\right) / \partial z_{n}$ has at least one zero point $\xi_{n}$ (i) on the side parallel to the $z_{n}$ axis. Noting that $\partial\left(\pi_{h} u_{i}\right) / \partial z_{n}$ is constant on $\tau_{j}$ yields that

$$
\nabla_{2, n}\left(u_{i}-\pi_{h} u_{i}\right)(z) \equiv \nabla_{2, n} u_{i}(z)-\nabla_{2, n} u_{i}\left(\xi_{n}\right)
$$

and hence the desired result (4.14) follows from the mean value theorem. 
If in addition $\mathbf{u} \in\left[C^{2, \beta}(\bar{\Omega})\right]^{r}$, we have that

$$
\left|\nabla_{2, n}\left(\mathbf{u}-\pi_{h} \mathbf{u}\right)(z)\right| \leq C h\left|H_{2, n}[\mathbf{u}](z)\right|+C h^{1+\beta}, \quad n=1 \rightarrow 2 .
$$

Therefore from (3.3) with $\delta_{1}=0$ and performing a similar calculation as in Theorem 4.2a it follows that

$$
\begin{aligned}
\left|\mathbf{u}-\mathbf{u}^{h}\right|_{\left[W^{1, p}\left(\Omega^{h}\right)\right]^{r}}^{2} & \leq C \sum_{n=1}^{2} \int_{\Omega^{h}}\left[\left|\nabla_{2, n} \mathbf{u}\right|+\left|\nabla_{2, n}\left(\mathbf{u}-\pi_{h} \mathbf{u}\right)\right|\right]^{p^{+}-2}\left|\nabla_{2, n}\left(\mathbf{u}-\pi_{h} \mathbf{u}\right)\right|^{2} d \Omega^{h} \\
& \leq\left. C h^{2} \sum_{n=1}^{2} \int_{\Omega^{h}}\left|\nabla_{2, n} \mathbf{u}\right|\right|^{p^{+}-2}\left|H_{2, n}[\mathbf{u}]\right|^{2} d \Omega^{h}+C h^{p^{+}(1+\beta)} .
\end{aligned}
$$

The desired result (4.13a) then follows from (4.12). The result (4.13b) follows from (4.13a) and Lemma 4.1 with $\alpha=p^{+}-2, \mathbf{w} \equiv \nabla_{2, n} \mathbf{u}$, and hence $\nabla \mathbf{w} \equiv H_{2, n}[\mathbf{u}]$.

We can also establish the analogue of Theorem $4.2 \mathrm{~b}$ for this case. However, it is not clear if the result in [15] can be generalised to this case, that is, if $W^{1+2 / p^{+}, p^{+}}(\Omega)$ regularity of $\mathbf{u}$ can guarantee the optimal error bounds in Theorem 4.3. In addition, very little is known about the regularity of solutions in this case.

Remark 4.3. As previously mentioned, all the proofs and results of $\S \S 1-3$ hold for higherdimensional problems and more general problems in which the $k$ appearing in $K_{n}, n=1 \rightarrow m$, (see (1.3c)) may be different but still satisfies Assumptions (A) or (B) for the same $p$. However, some of the results in $\S 4$ hold only for the two-dimensional case. This is mainly due to Sobolev embedding and regularity results. It is also worth noting that $\nabla \mathbf{u}$ in (1.3) can be replaced, for example, by the rate of deformation tensor $D(\mathbf{u})$ (see (1.4)).

Instead of working on a concrete form of $K$ as in (1.3), one could alternatively state some abstract structural conditions for $K$. Let us briefly examine the case $m=1$. To obtain Theorem 3.1 one needs only the following assumptions on $K$.

Assumption (AK): There exist constants $p \in(1,2]$ and $C, M>0$ such that for any $x, y \in R^{2 \times r}$

(AK1) $|K(x) x-K(y) y| \leq C|x-y|(|x|+|y|)^{p-2}$,

(AK2) $(K(x) x-K(y) y, x-y) \geq M|x-y|^{2}(1+|x|+|y|)^{p-2}$;

or

Assumption (BK): There exist constants $p \in[2, \infty)$ and $C, M>0$ such that for any $x, y \in R^{2 \times r}$

(BK1) $|K(x) x-K(y) y| \leq C|x-y|(1+|x|+|y|)^{p-2}$,

(BK2) $(K(x) x-K(y) y, x-y) \geq M|x-y|^{2}(|x|+|y|)^{p-2}$.

Then the rest of work is a matter of proving suitable regularity for the solutions. The system (1.3) certainly satisfies these conditions in the case $m=1$ if $k$ satisfies Assumptions (A) or (B) (proving this is actually the main task of $\S 3$ ). This formulation includes some non-Uhlenbeck type systems too. This approach also leads to a compact presentation. It does not, however, include the system (1.2) as it stands and seems only to cover systems essentially having the same degenerate nature as that of (1.1). However, it can be adapted to include (1.2), but in our opinion much more work is needed in order to find good general structural conditions which cover a broad class of degenerate elliptic systems, i.e., problems which contain a mixture of the type of nonlinearities in (1.1) and (1.2).

We now examine the case $p \in[2, \infty)$. From (3.11b) and (4.1) it follows that $\| \mathbf{u}-$ $\mathbf{u}^{h} \|_{\left[W^{1, p}\left(\Omega^{h}\right)\right]^{r}} \leq C h^{2 / p}$ if $\left[u \in W^{2, p}(\Omega)\right]^{r}$, which is the best existing result in the literature (see $[6])$. As we have seen, however, $u$ is rarely in $\left[W^{2, p}(\Omega)\right]^{r}$; see $(4.3 \mathrm{c})$ and $(4.5 \mathrm{~d})$. From (3.11c) and (4.1) we see that this convergence rate is still maintained if $\mathbf{u} \in\left[H^{2}(\Omega) \cap W^{1, \infty}(\Omega)\right]^{r}$, a much weaker regularity restriction on $\mathbf{u}$ and achievable (see (4.4d)). However, the convergence rate $h^{2 / p}$ degenerates as $p \rightarrow \infty$. 
One can easily improve on this result if $k$ is not degenerate; that is, $k$ satisfies (B) for a $p \in[2, \infty)$ and $(\mathrm{B} 2)$ for $p^{-}=2$. We have the following result.

THEOREM 4.4. Let $k$ satisfy (B) for a $p \in[2, \infty)$ and (B2) for $p^{-}=2$. Let $\mathbf{u}$ and $\mathbf{u}^{h}$ be the unique solutions of (WP) and (WP) ${ }^{h}$. If $\mathbf{u} \in\left[W^{2, q}(\Omega) \cap W^{1, \infty}(\Omega)\right]^{r}$ for a $q \in[1,2]$, then

$$
\left\|\mathbf{u}-\mathbf{u}^{h}\right\|_{\left[H^{1}\left(\Omega^{h}\right)\right]^{r}}^{2} \leq C h^{q} .
$$

Proof. The result follows directly from (3.11c) and (4.1).

For example, Theorem 4.4 applies to $k(t) \equiv\left(1+t^{2}\right)^{(p-2) / 2}$ with $p \in[2, \infty)$. We note that the results of [12] and [11] under stronger assumptions also apply in this case. We now consider the degenerate case.

Example 4.4. Let $r=m=1$ and $k(t) \equiv t^{p-2}, p \in(2, \infty)$. Consider the continuous piecewise linear finite element approximation on a uniform mesh of the one-dimensional version of (WP) with $\Omega=(-1,1), u(-1)=u(1)=0$, and $f \equiv 1$. It is easy to establish the following results:

$$
\begin{aligned}
\left\|u-u^{h}\right\|_{W^{1,1}(\Omega)} & \leq C h \quad \text { if } p>2, \\
C_{1} h^{(2 p-1) / p(p-1)} & \geq\left\|u-u^{h}\right\|_{W^{1, p}(\Omega)} \\
& \geq C_{2}\left\|u-\pi_{h} u\right\|_{W^{1, p}(\Omega)} \geq C_{3} h^{(2 p-1) / p(p-1)} \quad \text { if } p \geq(3+\sqrt{ } 5) / 2 .
\end{aligned}
$$

We see from this example that one cannot expect $u^{h}$ to converge to $u$ at the optimal rate $h$ in the $W^{1, p}\left(\Omega^{h}\right)$ norm, as can be expected from the restricted regularity of $u$ (see (4.3c)). We also see how the convergence rate degenerates as $p \rightarrow \infty$, in agreement with (3.4) and $(3.11 \mathrm{~b}-\mathrm{c})$. However, one might hope to prove that $u^{h}$ convergences to $u$ at the optimal rate $h$ in the $W^{1,1}\left(\Omega^{h}\right)$ norm and this can indeed be proven under the restriction $|f|^{-\gamma} \in L^{1}(\Omega)$ for a suitable $\gamma>0$ by further exploring (3.4b). We do not, however, go into the details here; see [4] for the case $r=m=1$ and $k(t) \equiv t^{p-2}, p \in(2, \infty)$. The case $m=2$ is more complicated, although some similar results can still be obtained.

\section{REFERENCES}

[1] C. AtKINSON AND C. R. CHAMPION, Some boundary value problems for the equation $\nabla \cdot\left(|\nabla \phi|^{N} \nabla \phi\right)=0$, Quart. J. Mech. Appl. Math., 37 (1984), pp. 401-419.

[2] C. ATKINSON AND C. W. JoNES, Similarity solutions in some non-linear diffusion problems and in boundarylayer flow of a pseudo plastic fluid, Quart. J. Mech. Appl. Math., 27 (1974), pp. 193-211.

[3] J. BARANGER AND K. NAJIB, Numerical analysis of quasi-Newtonian flow obeying the power law or the Carreau law, Numer. Math., 58 (1990), pp. 35-49.

[4] J. W. BARRETT AND W. B. LiU, Finite element approximation of the p-Laplacian, Math. Comp., 61 (1993), pp. 523-537.

[5] - Finite element error analysis of a quasi-Newtonian flow obeying the Carreau or power law, Numer. Math, 64 (1993), pp. 433-453.

[6] S. S. CHow, Finite element error estimates for non-linear elliptic equations of monotone type, Numer. Math., 54 (1988), pp. 373-393.

[7] - Finite element error estimates for a blast furnace gas flow problem, SIAM. J. Numer. Anal., 29 (1992), pp. 769-780.

[8] P. G. Ciarlet, M. H. SChultz, AND R. S. VARGa, Numerical methods of high order accuracy for nonlinear boundary value problems: V. Monotone operator theory, Numer. Math., 13 (1969), pp. 51-77.

[9] P. G. Ciarlet, The Finite Element Method for Elliptic Problems, North-Holland, Amsterdam, 1978.

[10] F. H. Clarke, Optimization and Nonsmooth Analysis, Wily-Interscience, New York, 1983.

[11] M. DOBROWOLSKI AND R. RANNACHER, Finite element methods for nonlinear elliptic systems of second order, Math. Nachr., 94 (1980), pp. 155-172.

[12] J. FREHSE AND R. RANNACHER, Asymptotic $L^{\infty}$-error estimates for linear finite element approximations of quasilinear boundary problems, SIAM. J. Numer. Anal., 15 (1978), pp. 419-431.

[13] R. GLOWINSKI AND A. MARROCCO, Sur l'approximation par elements finis d'ordre un, et la resolution, par penalisation-dualite, d' une classe de problemes de Dirichlet non lineaires, R.A.I.R.O. Módel. Math. Anal. Numér., 2 (1975), pp. 41-64. 
[14] W. B. LIU AND J. W. BARRETT, A remark on the regularity of the solutions of the p-Laplacian and its applications to their finite element approximation, J. Math. Anal. Appl., 178 (1993), pp. 470-487.

[15] - A further remark on the regularity of the solutions of the p-Laplacian and its applications to their finite element approximation, J. Nonlinear. Anal., 21 (1993), pp. 379-387.

[16] - Higher order regularity for the solutions of some degenerate quasilinear elliptic equations, SIAM J. Math. Anal., 24 (1993), pp. 1522-1536.

[17] M. A. Noor, Finite element approximation theory for strong nonlinear problems, Comment. Math. Univ. St. Paul, 31 (1982), pp. 1-7.

[18] J. R. PhILIP, N-diffusion, Aust. J. Phys., 14 (1961), pp. 1-13.

[19] J. R. RiCE AND G. F. RoSENGREN, Plane strain deformation near a crack tip in a power law hardening material, J. Mech. Phys. Solids., 16 (1968), pp. 1-12.

[20] J. R. RICE, Mathematical analysis in the mechanics of fracture. Fracture-an Advanced Treatise, Vol. II., H. Liebowitz, ed., Academic Press, New York, 1968, pp. 191-308.

[21] P. TOLKSDORF, On the Dirichlet problem for quasilinear equations in domains with conical boundary points, Comm. Partial. Diff. Eqns., 8 (1983), pp. 773-817.

[22] V. B. TyukHTIN, The rate of convergence of approximation methods of one-sided problems I, Vestnik Leningrad Univ. Mat. Mekh. Astronom. 13 (1982), pp. 111-113 (in Russian). 